\title{
High HIV incidence in men who have sex with men following an early syphilis diagnosis: is there room for pre-exposure prophylaxis as a prevention strategy?
}

\author{
Nicolò Girometti, ${ }^{1,2}$ Angela Gutierrez, ${ }^{2}$ Nneka Nwokolo, ${ }^{2}$ Alan McOwan, ${ }^{2}$ \\ Gary Whitlock ${ }^{2}$
}

1 Infectious Diseases Unit, S. Orsola Malpighi Hospital, University of Bologna, Bologna, Italy

${ }^{2}$ Chelsea \& Westminster Hospital NHS Foundation Trust, London, UK

\section{Correspondence to} Dr Nicolò Girometti, Chelsea \& Westminster Hospital NHS Foundation Trust, 56 Dean Street, London W1D 6AQ, UK; nicogir@hotmail.it

These data were previously presented at the BASHH conference, Oxford 10-12 July 2016.

Sex Transm Infect 2016;92(Suppl 1):P172.

Received 25 August 2016 Revised 24 September 2016 Accepted 29 September 2016 Published Online First 19 October 2016
ABSTRACT

Objectives HIV pre-exposure prophylaxis (PrEP) is becoming a pivotal strategy for HIV prevention. Understanding the impact of risk factors for HIV transmission to identify those at highest risk would favour the implementation of PrEP, currently limited by costs. In this service evaluation, we estimated the incidence of bacterial STIs in men who have sex with men (MSM) diagnosed with early syphilis attending a London sexual health clinic according to their HIV status. In addition, we estimated the incidence of HIV infection in HIV-negative MSM, following a diagnosis of early syphilis.

Methods We undertook a retrospective case note review of all MSM patients diagnosed with early syphilis between January and June 2014. A number of sexual health screens and diagnoses of chlamydia, gonorrhoea and HIV were prospectively analysed following the syphilis diagnosis.

Results 206 MSM were diagnosed with early syphilis. $110(53 \%)$ were HIV-negative at baseline, 96 (47\%) were HIV-positive. Only age (37 vs 32 years, $p=0.0005$ ) was significantly different according to HIV status of MSM at baseline. In HIV-negative versus HIV-positive MSM, incidence of rectal chlamydia infection at followup was 27 cases vs 50/100 person-years of follow-up (PYFU) ( $p=0.0039), 33$ vs 66/100 PYFU ( $p=0.0044)$ for rectal gonorrhoea and 10 vs $26 / 100$ PYFU ( $p=0.0044)$ for syphilis reinfection, respectively. Total follow-up for 110 HIV-negative MSM was 144 person-years. HIV incidence was 8.3/100 PYFU (Cl 4.2 to 14).

Conclusions $A$ diagnosis of early syphilis carries a high risk of consequent HIV seroconversion and should warrant prioritised access to prevention measures such as PrEP and regular STI screening to prevent HIV transmission.

\section{INTRODUCTION}

HIV pre-exposure prophylaxis (PrEP) along with treatment as prevention is becoming the mainstay of combination strategies for HIV prevention. ${ }^{1}$ PrEP has been shown to be $86 \%$ effective in reducing HIV acquisition in men who have sex with men (MSM) in two large trials, ${ }^{23}$ and understanding how to better identify high-risk MSM who should receive PrEP could improve its costeffectiveness, which appears to be the major obstacle to a widespread implementation. ${ }^{45}$
Recent acquisition of certain STIs is associated with increased HIV incidence in MSM. ${ }^{6}$ In particular, rectal chlamydia or gonorrhoea infection can increase the risk of HIV seroconversion up to eight times. ${ }^{7}$ Syphilis infection has also been found to be an independent risk factor for future HIV acquisition. ${ }^{8-10}$ Data on real-life practice ${ }^{10}$ confirm the increased risk of HIV acquisition with rectal STIs and syphilis, previously demonstrated in controlled studies, mostly where PrEP efficacy was the primary end point. Nonetheless, studies performed in a National Health Service context are lacking.

UK national guidance on the management of syphilis recommends that all people with syphilis be offered sexual health screening including HIV testing. ${ }^{11}$ This service evaluation assesses the HIV and STI rates in people presenting with syphilis. We estimate the incidence of bacterial STIs in MSM diagnosed with early syphilis, according to their HIV status. In addition, we calculate the incidence of HIV infection in HIV-negative MSM following a diagnosis of early syphilis.

\section{METHODS}

The study was conducted at 56 Dean Street (56DS), a large HIV and sexual health clinic in London, UK.

All MSM, irrespective of their baseline HIV status, who received a diagnosis of primary, secondary or early latent syphilis between January and June 2014, were identified using our electronic database. We undertook a retrospective case note review of patient records and extracted data on subsequent management. Numbers of sexual health screens and Chlamydia trachomatis (CT), Neisseria gonorrhoeae (GC), HIV, hepatitis B virus (HBV) and hepatitis $\mathrm{C}$ virus (HCV) diagnoses were prospectively analysed. Samples for gonorrhoea and chlamydia testing were processed by nucleic acid amplification tests. Syphilis diagnosis was serological or clinical (confirmed by PCR on swab or serology), and the serological response was subsequently monitored. All patients who were HIV-negative were offered HIV test at baseline, according to the risk: third generation point-of-care test or fourth generation serology. Those resulting positive for either baseline HIV test were considered in the group of patients who 
were HIV-positive. At follow-up, incident syphilis was defined as at least a fourfold increase in Rapid plasma reagin test (RPR) titre from prior test and documentation of prior treatment appropriate for the stage of syphilis. All individuals received treatment in accordance with the BASHH guidelines. ${ }^{11}$ We used the following tests to measure the differences between baseline characteristics according to HIV status: Mann-Whitney U test (age and number of sexual partners), $\chi^{2}$ tests (syphilis stage), Z-test (penicillin treatment, drug use). We calculated $95 \%$ exact CIs and $\mathrm{p}$ values for incidence rates using Poisson distribution. This evaluation assesses current practice using existing data, and as such ethical approval was not sought.

\section{RESULTS}

A total of 206 MSM were diagnosed with early syphilis between January and June 2014: primary $(64,31 \%)$, secondary (45, $22 \%$ ), early latent $(97,47 \%)$. Their median age was 35 (IQR: $29-41$ years), with a median number of sexual partners in the previous 3 months of 4 (IQR: 2-8). The majority (191,93\%) received treatment with 2.4 Millions of units (MU) benzathine penicillin intramuscularly. A total of 87 (42\%) had reported recreational drug use during sexual activity in the previous 3 months; 110 (53\%) were HIV-negative at baseline, 96 (47\%) were HIV-positive $(n=93)$ or were diagnosed with HIV at the time of syphilis diagnosis $(\mathrm{n}=3)$ (see table 1 ).

Three patients were HIV-HCV coinfected, one monoinfected $\mathrm{HCV}$ and two monoinfected HBV at baseline. HIV-positive MSM with early syphilis were older than HIV-negative MSM (37 vs 32 years, $\mathrm{p}=0.0005)$. Otherwise, there were no differences in baseline characteristics according to the HIV status of MSM.

According to HIV status at baseline, subsequent incidence of rectal chlamydia infection was respectively 27 cases in HIV-negative vs 50 in HIV-positive/100 person-years follow-up

Table 1 Characteristics of patients with early syphilis according to their baseline HIV status.

\begin{tabular}{|c|c|c|c|}
\hline n (\%) & $\begin{array}{l}\text { Syphilis in } \\
\text { HIV-positive } \\
n=96(47 \%)\end{array}$ & $\begin{array}{l}\text { Syphilis in } \\
\text { HIV-negative } \\
n=110(53 \%)\end{array}$ & p Value \\
\hline Age, median years (IQR) & $37(33-42)$ & $32(28-40)$ & 0.0005 \\
\hline Syphilis stage & & & 0.57 \\
\hline Primary (\%) & $30(31)$ & $34(31)$ & \\
\hline Secondary (\%) & $18(19)$ & $27(25)$ & \\
\hline Early latent (\%) & $48(50)$ & $49(45)$ & \\
\hline $\begin{array}{l}\text { Treatment with benzathine } \\
\text { penicillin }(\%)\end{array}$ & $86(90)$ & $105(95)$ & 0.11 \\
\hline $\begin{array}{l}\text { Median number of sexual } \\
\text { partners in last } 3 \text { month (IQR) }\end{array}$ & $4(2-9)$ & $4(2-7)$ & 0.91 \\
\hline Reported drug use (\%) & $45(47)$ & $42(38)$ & 0.21 \\
\hline Mephedrone (\%) & $32(33)$ & $33(30)$ & \\
\hline GHB/GBL (\%) & $27(28)$ & $24(22)$ & \\
\hline Crystal metamphetamine (\%) & $28(29)$ & $20(18)$ & \\
\hline Intravenous drug use (\%) & $18(19)$ & $17(15)$ & \\
\hline $\begin{array}{l}\text { Rectal chlamydia infection } \\
\text { (incidence/100 PYFU, Cl) }\end{array}$ & $50(36-68)$ & $27(19-36)$ & 0.004 \\
\hline $\begin{array}{l}\text { Rectal gonorrhoea infection } \\
\text { (incidence/100 PYFU, CI) }\end{array}$ & $66(50-87)$ & $33(25-44)$ & 0.004 \\
\hline $\begin{array}{l}\text { Syphilis reinfection } \\
\text { (incidence/100 PYFU, CI) }\end{array}$ & $26(16-40)$ & $10(5.7-17)$ & 0.004 \\
\hline $\begin{array}{l}\text { HIV infection } \\
\text { (incidence/100 PYFU, CI) }\end{array}$ & $\mathrm{n} / \mathrm{a}$ & $8.3(4.2-14)$ & \\
\hline
\end{tabular}

(PYFU) ( $\mathrm{p}=0.004), 33$ vs 66/100 PYFU for rectal gonorrhoea $(\mathrm{p}=0.0044)$ and 10 vs $26 / 100$ PYFU for syphilis reinfection $(p=0.004)$. Until February 2016, total follow-up for 110 HIV-negative MSM was 144 person-years. Among these, $12(11 \%)$ developed a subsequent HIV infection, equating to an HIV incidence of $8.3 / 100$ PYFU (95\% CI 4.2 to 14). Of these, seven had a subsequent negative HIV test prior to their HIV diagnosis. Of the remaining five, the last negative HIV test was at baseline syphilis diagnosis. Of these, four were diagnosed more than 100 days later. One patient had a negative fourth generation HIV test at syphilis diagnosis and a subsequent positive p24 antigen 19 days later with symptoms of acute HIV infection.

\section{DISCUSSION}

Our study highlights early syphilis as a risk factor for HIV acquisition in MSM, predicting a HIV incidence of 8.3/100 PYFU in a real-life setting. Nearly half of the population presented here are regular users of recreational drugs during sex (chemsex). MSM with early syphilis who attend our service have high rates of subsequent bacterial rectal STIs and syphilis reinfection, irrespectively of HIV status.

Our data confirm those seen in controlled studies. Syphilis was an independent predictor of HIV acquisition (HR 4.67) in a cohort in San Diego, USA, although a subanalysis of risk factors for HIV in those infected with syphilis was not performed. ${ }^{9}$ Syphilis also emerged as an independent risk factor for HIV acquisition, although to a lesser extent, in similar studies of larger populations: Pathela $e \mathrm{al}^{10}$ found a 5.6/100 PYFU incidence of HIV in MSM in New York City, USA, while in iPrEX, Solomon $\mathrm{et}_{\mathrm{al}}^{8}$ found a 7.3/100 PYFU incidence. Here, the impact of PrEP in patients with a syphilis diagnosis was also measured.

As in other studies assessing the incidence of HIV following a diagnosis of STIs, our estimates of incidence may be underestimates, given the possibility that MSM in our cohort may have tested elsewhere. Also, our study findings may need to be contextualised for our clinical setting. Our sexual health clinic is located in Soho, London, and attracts a large proportion of London's MSM community: 56DS diagnoses 1 in 6 of the UK's HIV in MSM and serves a large number of sexually active HIV-positive individuals, as seen in nearly $50 \%$ of our early syphilis diagnoses.

Identifying key risk factors for HIV acquisition allows us to better target prevention strategies, particularly in a context of limited economic resources. Our HIV incidence following a diagnosis of early syphilis is comparable with the deferred arm of the PROUD study (9.0/100 person-years), ${ }^{2}$ and with the incidence following postexposure prophylaxis use (7.6/100 personyears). ${ }^{12}$ This suggests that intensive risk reduction and PrEP would be beneficial in HIV-negative MSM with early syphilis by reducing their risk of HIV acquisition.

In our population, high levels of subsequent bacterial rectal STIs, along with a rising problem of 'chemsex' use, suggest that the approach must be on multiple levels, supporting the inclusion of regular STI screening in PrEP management guidelines and chemsex support.

\section{Handling editor Jackie A Cassell}

Contributors NG and GW contributed to ideation, data collection, writing and analysis of this study. AG contributed with data collection. NN and AMcO contributed with the analysis and editing.

Competing interests None declared.

Provenance and peer review Not commissioned; externally peer reviewed. 


\section{REFERENCES}

1 Punyacharoensin N, Edmunds WJ, De Angelis D, et al. Effect of pre-exposure prophylaxis and combination HIV prevention for men who have sex with men in the UK: a mathematical modelling study. Lancet HIV 2016;3:e94-104.

2 McCormack S, Dunn DT, Desai M, et al. Pre-exposure prophylaxis to prevent the acquisition of HIV-1 infection (PROUD): effectiveness results from the pilot phase of a pragmatic open-label randomised trial. The Lancet 2016;387:53-60.

3 Molina JM, Capitant C, Spire B, et al., ANRS IPERGAY Study Group. On-demand preexposure prophylaxis in men at high risk for HIV-1 infection. N Engl J Med 2015;373:2237-46.

4 McManus J, Harrison D. Funding PrEP for HIV prevention. BMJ 2016;354:i3515.

5 Ross EL, Cinti SK, Hutton DW. Implementation and operational research: a costeffective, clinically actionable strategy for targeting HIV preexposure prophylaxis to high-risk men who have sex with men. J Acquir Immune Defic Syndr 2016;72:e61-7.

6 Zetola NM, Bernstein KT, Wong E, et al. Exploring the relationship between sexually transmitted diseases and HIV acquisition by using different study designs. J Acquir Immune Defic Syndr 2009;50:546-51.
7 Bernstein KT, Marcus JL, Nieri G, et al. Rectal gonorrhea and chlamydia reinfection is associated with increased risk of HIV seroconversion. J Acquir Immune Defic Syndr 2010;53:537-43.

8 Solomon MM, Mayer KH, Glidden DV, et al. Syphilis predicts HIV incidence among men and transgender women who have sex with men in a preexposure prophylaxis trial. Clin Infect Dis 2014;59:1020-6.

9 Hoenigl M, Green N, Mehta SR, et al. Risk factors for acute and early HIV infection among men who have sex with men (MSM) in San Diego, 2008 to 2014: a Cohort Study. Medicine (Baltimore) 2015;94:e1242.

10 Pathela P, Braunstein SL, Blank S, et al. The high risk of an HIV diagnosis following a diagnosis of syphilis: a population-level analysis of New York City men. Clin Infect Dis 2015;61:281-7.

11 Kingston $M$, French $P$, Higgins $S$, et al. UK national guidelines on the management of syphilis 2015. Int J STD AIDS 2016;27:421-46.

12 Whitlock G, McCormack C, Fearnley J, et al. High HIV incidence in men who have sex with men attending for postexposure prophylaxis: a service evaluation. Sex Transm Infect 2016;doi:10.1136/sextrans-2016-052623. 